Article

\title{
Investigation of Antifungal Mechanisms of Thymol in the Human Fungal Pathogen, Cryptococcus neoformans
}

\author{
Kwang-Woo Jung ${ }^{1, *}$, Moon-Soo Chung ${ }^{1}$, Hyoung-Woo Bai ${ }^{1,2} \oplus$, Byung-Yeoup Chung ${ }^{1}$ and Sungbeom Lee ${ }^{1,2, *}$ \\ 1 Radiation Research Division, Advanced Radiation Technology Institute, Korea Atomic Energy Research \\ Institute, Jeongeup-si 56212, Jeollabuk-do, Korea; mschung@kaeri.re.kr (M.-S.C.); hbai@kaeri.re.kr (H.-W.B.); \\ bychung@kaeri.re.kr (B.-Y.C.) \\ 2 Department of Radiation Science and Technology, University of Science and Technology, Daejeon 34113, \\ Yuseong-gu, Korea \\ * Correspondence: kwjung@kaeri.re.kr (K.-W.J.); sungbeom@kaeri.re.kr (S.L.)
}

check for

updates

Citation: Jung, K.-W.; Chung, M.-S.; Bai, H.-W.; Chung, B.-Y.; Lee, S.

Investigation of Antifungal

Mechanisms of Thymol in the Human

Fungal Pathogen, Cryptococcus

neoformans. Molecules 2021, 26, 3476.

https://doi.org/10.3390/

molecules 26113476

Academic Editors: Marcello Iriti and Md. Moshfekus Saleh-E-In

Received: 23 April 2021

Accepted: 3 June 2021

Published: 7 June 2021

Publisher's Note: MDPI stays neutral with regard to jurisdictional claims in published maps and institutional affiliations.

Copyright: (c) 2021 by the authors. Licensee MDPI, Basel, Switzerland. This article is an open access article distributed under the terms and conditions of the Creative Commons Attribution (CC BY) license (https:/ / creativecommons.org/licenses/by/ $4.0 /)$.

\begin{abstract}
Due to lifespan extension and changes in global climate, the increase in mycoses caused by primary and opportunistic fungal pathogens is now a global concern. Despite increasing attention, limited options are available for the treatment of systematic and invasive mycoses, owing to the evolutionary similarity between humans and fungi. Although plants produce a diversity of chemicals to protect themselves from pathogens, the molecular targets and modes of action of these plant-derived chemicals have not been well characterized. Using a reverse genetics approach, the present study revealed that thymol, a monoterpene alcohol from Thymus vulgaris L., (Lamiaceae), exhibits antifungal activity against Cryptococcus neoformans by regulating multiple signaling pathways including calcineurin, unfolded protein response, and HOG (high-osmolarity glycerol) MAPK (mitogen-activated protein kinase) pathways. Thymol treatment reduced the intracellular concentration of $\mathrm{Ca}^{2+}$ by controlling the expression levels of calcium transporter genes in a calcineurin-dependent manner. We demonstrated that thymol decreased $N$-glycosylation by regulating the expression levels of genes involved in glycan-mediated post-translational modifications. Furthermore, thymol treatment reduced endogenous ergosterol content by decreasing the expression of ergosterol biosynthesis genes in a HOG MAPK pathway-dependent manner. Collectively, this study sheds light on the antifungal mechanisms of thymol against $C$. neoformans.
\end{abstract}

Keywords: thymol; Cryptococcus neoformans; antifungal activity; calcium homeostasis; $N$-glycosylation; ergosterol

\section{Introduction}

The incidence rate of systemic and invasive mycoses caused by opportunistic fungal pathogens, including Candida albicans, Aspergillus fumigatus, and Cryptococcus neoformans, has increased over the past decades due to the extension of human lifespan and increase in the number of immunocompromised patients with organ transplantation. Recently, it has been estimated that there are more than 4,000,000 infections caused by opportunistic fungal pathogens annually [1]. Despite global concerns, therapeutic options are limited due to the evolutionarily similar cellular structures observed in both humans and fungi [2,3]. In addition, given that antifungal agents such as azole drugs, polyene drugs, and flucytosine have adverse effects [4], there is an urgent need for the identification of novel compounds and targets for treatment of this condition, for the betterment of public health.

Plants produce a large number of chemicals, some of which are used as primary metabolites, while others are used to protect themselves from pathogens and herbivores. Of the different plant defense compounds, phytoanticipins and phytoalexins have been widely characterized over several decades [5]. Phytoanticipins are pre-existing compounds found in diverse locations that are released from organelles immediately after a pathogen attack. 
In contrast, phytoalexins are metabolites that are synthesized de novo and accumulate following pathogen attack [6].

Terpene, a hydrocarbon compound constructed from five-carbon isoprene, is classified as a monoterpene (C10), sesquiterpene (C15), and diterpene (C20), based on the isoprene units. Terpenoids are organic chemicals derived from terpenes, with additional oxygen-containing functional group(s). Thyme oil (from Thymus vulgaris L., Lamiaceae) consists of diverse monoterpenoids, including thymol (10-64\%), carvacrol (0.4-20.6\%), and $p$-cymene $(9.1-22.2 \%)$ and is used as a food additive as well as for pharmacological and cosmetic purposes [7]. Thymol, a main component of thyme oil, exhibits a variety of biological activities, such as anti-carcinogenesis and anti-inflammatory activities [8]. Thymol also exhibits antibacterial activity against an array of Gram-positive and Gram-negative strains $[9,10]$. In addition to its antibacterial effect, it exhibits fungicidal activity against Candida albicans, Botrytis cinerea, Rhizopus oryzae, and Aspergillus species [11-14]. Recently, Teixeira et al. analyzed the minimum inhibitory concentration and minimum fungicidal concentration values of thymol against 10 clinical strains of $C$. neoformans [15]. However, there is limited information on its mechanism of action.

C. neoformans is responsible for fatal meningoencephalitis in immunocompromised individuals and annually causes more than 200,000 infections globally [16]. Infectious Cryptococcus propagules are inhaled through the respiratory tract of the host. It then disseminates to the brain via the bloodstream and infects the central nervous system by crossing the blood-brain barrier [17,18]. Due to the availability of its genetic mutants and molecular tools, C. neoformans has become a model species to study fungal pathogenicity and virulence mechanisms [19]. In this study, using a reverse genetic approach, we found that thymol not only induced intracellular calcium imbalance in C. neoformans but also inhibited $\mathrm{N}$-glycosylation modification of the Cpy protein. In addition, thymol treatment negatively affected ergosterol biosynthesis.

Collectively, this study provides insight into the antifungal mechanisms of C. neoformans modulated by natural compounds such as thymol. Furthermore, it suggests that thymol can be used as an antifungal agent or a putative supplement to currently available antibiotics, which would reduce the chance of overuse of antibiotics.

\section{Results}

\subsection{Identification of Thymol-Responsive Signaling Pathways in C. neoformans}

Although thyme essential oil extracted from Thymus vulgaris L., (Lamiaceae) is known for its antifungal activity against human fungal pathogens $[11,12,15]$, information on alterations in signaling pathways and cellular changes within the pathogen following treatment with thyme oil remains unknown. First, we screened loss-of-function strains of C. neoformans, which are mutated in stress-responsive signaling pathways, for their viability post treatment with thyme oil constituents such as thymol, carvacrol, and $p$-cymene. Briefly, HOG1 encodes a high osmolarity glycerol mitogen-activated protein kinase (HOG MAPK) and is involved in the osmotic and oxidative stress response [20]. An Mpk1 kinase is a core protein of pathway for the cell wall [21]. Cna1 and Cnb1, catalytic and regulatory subunits of the calcineurin pathway, respectively, control intracellular calcium homeostasis [22]. Ire1 is a sensor kinase of unfolded protein response (UPR) and required for endoplasmic reticulum (ER) stress [23]. Cac1 (adenylyl cyclase) and Ras1 (a small GTPase) are upstream regulators of protein kinase $\mathrm{A}$ and are associated with growth and differentiation $[24,25]$. Rad53 controls DNA damage response by regulating the expression of DNA repair genes [26,27]. Spotting assays revealed that the $c n a 1 \Delta, c n b 1 \Delta$, and ire $1 \Delta$ mutants were sensitive to treatment with thymol and carvacrol, but not with $p$-cymene (Figure 1A). Furthermore, the hog $1 \Delta$ mutant exhibited more sensitivity to thymol, as compared to the wild type (WT). However, the growth inhibition effects of thymol, carvacrol, and $p$-cymene on the $\operatorname{cac} 1 \Delta, m p k 1 \Delta$, ras1 $\Delta$, and rad53 $\Delta$ mutants were negligible when compared to that on the WT (Figure 1A). 


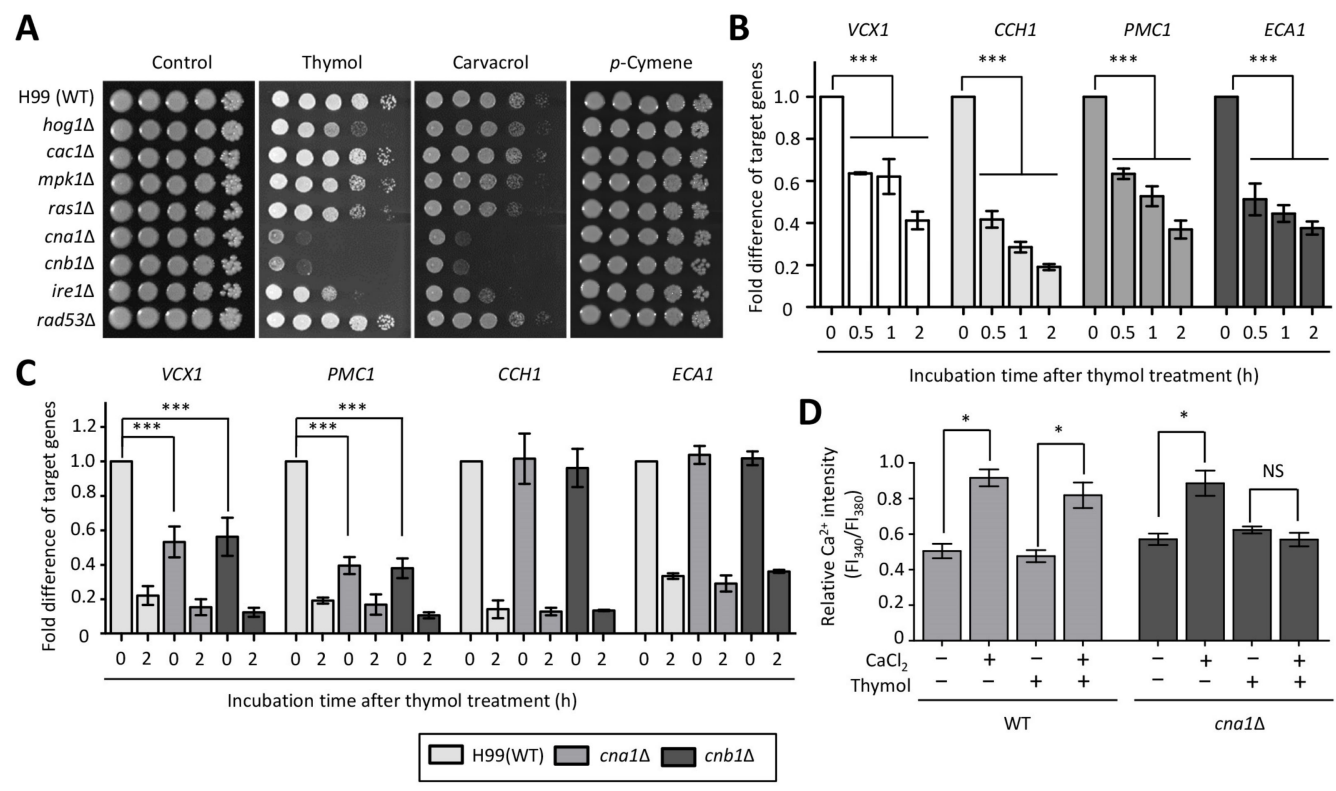

Figure 1. The calcineurin pathway is required for thymol resistance. (A) Growth inhibition of C. neoformans signaling mutants to monoterpenoid compounds that originate from thyme oil, including thymol, $p$-cymene, and carvacrol. (B,C) Expression levels of genes involved in calcium transport were determined using quantitative reverse transcription polymerase chain reaction (qRT-PCR) analysis with cDNA synthesized from total RNA isolated from the WT (H99) strain (B) and the indicated strains $(C)$ treated with thymol $(1 \mathrm{mM})$ during the indicated time-points. (D) Relative levels of intracellular Ca ${ }^{2+}$ concentration in the WT and cna1 $\Delta$ mutant upon thymol treatment. The fold differences of target genes were statistically analyzed using the Bonferroni's multiple comparison test and relative $\mathrm{Ca}^{2+}$ intensity was statistically analyzed using the Bonferroni's selected comparison test performed with Prism software $\left({ }^{*} p<0.05,{ }^{* *} p<0.01,{ }^{* *} p<0.001\right.$, and NS non-significant).

\subsection{The Effect of Thymol Treatment on the Intracellular $\mathrm{Ca}^{2+}$ Balance in C. neoformans}

Given the fact that carvacrol, a derivative of $p$-cymene that shows structural similarity with thymol, disrupts cellular $\mathrm{Ca}^{2+}$ homeostasis in S. cerevisiae [28] and that the relative abundance of calcium transporter genes is altered in an intracellular calcium-dependent manner [29], we determined the expression levels of the transporter genes upon thymol treatment. There was a gradual reduction in the expression levels of VCX1 (a vacuolar $\mathrm{Ca}^{2+}$-exchanger), CCH1 (a voltage-gated $\mathrm{Ca}^{2+}$-channel), PMC1 (a putative vacuolar $\mathrm{Ca}^{2+}$-ATPase), and ECA1 (a sarcoplasmic reticulum/ER $\mathrm{Ca}^{2+}$-ATPase) genes in the presence of thymol (Figure 1B). These results indicate that thymol also influences $\mathrm{Ca}^{2+}$ homeostasis in C. neoformans. These findings led us to monitor the expression levels of these genes in the $c n a 1 \Delta$ and $c n b 1 \Delta$ mutants. We found that a mutation per se of either CNA1 or CNB1 resulted in reduced expression levels of $V C X 1$ and $P M C 1$, as compared to those in the WT, at the basal level (Figure 1C), while thymol treatment resulted in no significant difference in gene expression levels between the WT and calcineurin mutants. Furthermore, the expression patterns of $\mathrm{CCH} 1$ and ECA1 in calcineurin mutants were similar to those in the WT, with or without thymol treatment (Figure 1C). Next, we measured the relative intracellular $\mathrm{Ca}^{2+}$ changes with FURA-2-acetoxymethyl ester (FURA-2-AM) staining post thymol treatment. We found that the relative intracellular $\mathrm{Ca}^{2+}$ levels in cells treated with thymol were not distinguishable from those in untreated cells (Figure 1D). Furthermore, the difference in intracellular $\mathrm{Ca}^{2+}$ levels was also similar between cells treated with thymol in the presence or absence of $\mathrm{CaCl}_{2}$. Since the expression levels of $V C X 1$ and PMC1 were reduced in both the cna1 $\Delta$ and $c n b 1 \Delta$ mutants, we compared the intracellular $\mathrm{Ca}^{2+}$ levels between calcineurin mutants and WT. Similar to WT, the relative intracellular $\mathrm{Ca}^{2+}$ levels remained unchanged in the cna1 $\Delta$ mutant post thymol treatment. However, the relative intracellular $\mathrm{Ca}^{2+}$ levels in the cna1 $\Delta$ mutant did not increase when treated with both $\mathrm{CaCl}_{2}$ and thymol, 
as compared to those in the WT. These data indicate that thymol-mediated intracellular $\mathrm{Ca}^{2+}$ levels are regulated in a calcineurin-dependent manner.

Crz1 is a well-known downstream transcription factor in the calcineurin pathway that is dephosphorylated in response to external stress [30]. To investigate the involvement of Crz1 in the mechanism of action of thymol, we monitored the dephosphorylation status of Crz1 upon thymol treatment. Crz1 was not dephosphorylated upon treatment with thymol (Figure 2A), coinciding with the observation that the $c r z 1 \Delta$ mutant exhibited a similar level of thymol resistance as the WT (Figure 2B). To further demonstrate that Crz1 is dispensable for thymol resistance, we constructed cna1 $1 \Delta r z 1 \Delta$ and $c n b 1 \Delta \operatorname{crz} 1 \Delta$ double mutants and monitored thymol resistance using these strains. In agreement with the result of Crz1 dephosphorylation, both $c n a 1 \Delta \operatorname{crz} 1 \Delta$ and $c n b 1 \Delta c r z 1 \Delta$ mutants were resistant to thymol, similar to the cna1 $\Delta$ and cnb1 $\Delta$ mutants (Figure 2B). Taken together, the calcineurin pathway modulates thymol resistance in a Crz1-independent manner.

A

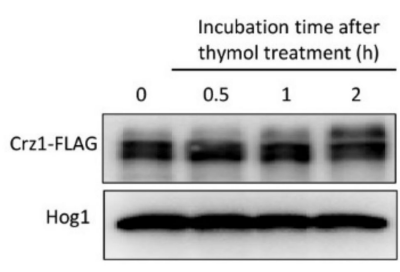

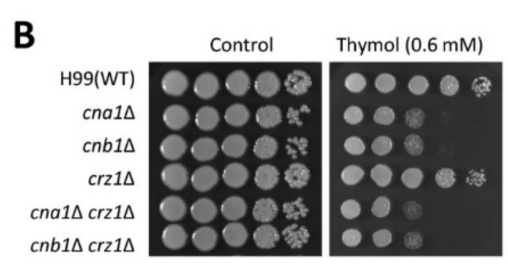

Figure 2. Calcineurin pathway is involved in thymol resistance in a Crz1-independent manner. (A) Phosphorylation of Crz1 in response to thymol. Total proteins were extracted from Crz1-FLAG strains treated with or without thymol $(1 \mathrm{mM})$ for $2 \mathrm{~h}$. The electrophoretic mobility of Crz1 was monitored using an anti-FLAG antibody. Anti-Hog1 polyclonal antibody was used as a loading control. (B) Crz1 was found to be not involved in thymol resistance in C. neoformans. The overnightcultured C. neoformans strains were serially diluted and spotted onto a yeast-peptone-dextrose (YPD) plate containing the indicated concentration of thymol. Cells were further incubated at $30^{\circ} \mathrm{C}$ and photographed daily for 3 days.

\subsection{The Effect of Thymol on the ER Stress in C. neoformans}

In addition to the calcineurin pathway, perturbation of the UPR pathway resulted in reduced growth upon thymol treatment (Figure 1A). Given that the Cryptococcus UPR pathway is activated by splicesome-independent splicing of HXL1 in an Ire1-dependent manner [23], we examined whether HXL1 splicing occurs during thymol treatment. As expected, the HXL1 splicing event occurred post thymol treatment (Figure 3A and Figure S2A). However, the $h x l 1 \Delta$ mutant showed WT levels of thymol resistance, although deletion of Ire1, an upstream factor, rendered cells susceptible to thymol. Furthermore, the ire $1 \Delta h x l 1 \Delta$ double mutant was resistant to thymol, similar to the ire $1 \Delta$ mutant (Figure 3B). Since the Cryptococcus UPR pathway regulates the expression levels of genes related to molecular chaperones (e.g., KAR2 encoding ER-resident molecular chaperone), protein degradation (e.g., DER1 encoding ER membrane protein involved in ER-associated degradation), vesiclemediated transport protein (e.g., ERV29 encoding proteins involved in vesicle formation), and protein glycosylation in an Hxl1-independent and -dependent manner [23], we measured the expression levels of the downstream subset in the presence of thymol. However, the expression levels of KAR2, DER1, and ERV29 did not change in response to thymol treatment (Figure S2B). Interestingly, expression of PMTs (encoding O-mannosyltransferases involved in $\mathrm{O}$-linked glycosylation) and $A L G 7$ (encoding UPD- $N$-acetyl-glucosamine-1-P transferase involved in $\mathrm{N}$-linked glycosylation) gradually decreased with thymol treatment (Figure 3C). These results imply that thymol affects the glycan-dependent protein modification. Furthermore, we monitored a glycosylation pattern of Cpy (vacuolar carboxypeptidase $\underline{\mathrm{Y}}$ ), which undergoes $\mathrm{N}$-glycosylation in the ER and is used as an indicator of protein glycosylation [31]. To observe glycosylation patterns using immunoblotting, we constructed strains containing Cpy (CNAG_06640) fused with a $4 \times$ FLAG epitope in the Cterminal region. Supporting the decrease in $A L G 7$ expression, we found that glycosylation 
of Cpy was reduced in the presence of thymol, indicating that thymol induces ER stress through inhibition of glycosylation (Figure 3D and Figure S2C).

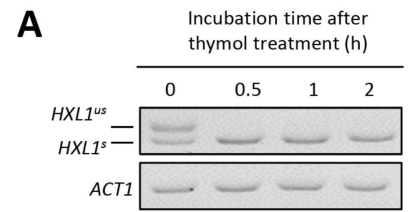

C

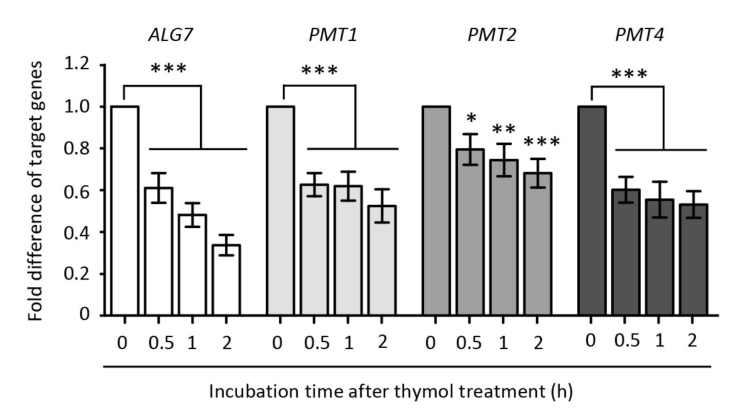

B
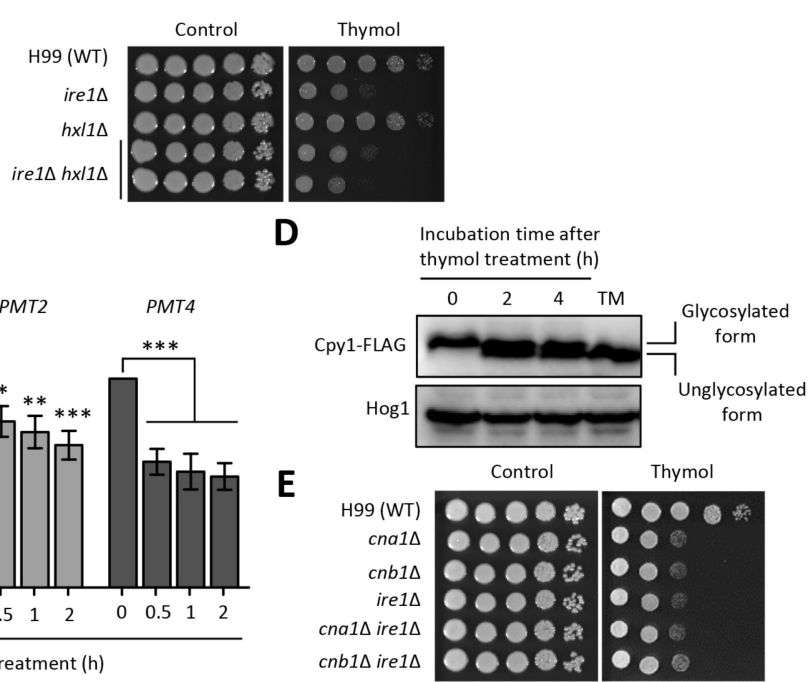

Figure 3. Thymol induces ER stress through reduction of protein glycosylation. (A) RT-PCR analysis of HXL1 splicing upon thymol treatment. HXL1 $1^{u s}$ and $H X L 1^{s}$ indicate the unspliced and spliced versions of HXL1, respectively. (B) Ire1 controls thymol resistance in an Hxl1-independent manner. (C) Quantitative RT-PCR analysis of UPR downstream genes upon thymol treatment. Fold difference in the expression of the target genes was statistically analyzed using the Bonferroni's multiple comparison test performed with Prism software $\left({ }^{*} p<0.05,{ }^{* *} p<0.01\right.$, and $\left.{ }^{* * *} p<0.001\right)$. (D) The glycosylation level of Cpy1 was reduced upon thymol treatment. TM is used as an inhibitor of protein glycosylation. Anti-Hog1 polyclonal antibody was used as a loading control. (E) The overlapping roles in thymol resistance between calcineurin and UPR pathways. TM, tunicamycin.

To investigate the relationship between the calcineurin and UPR pathways, we constructed $c n a 1 \Delta$ ire $1 \Delta$ and $c n b 1 \Delta$ ire $1 \Delta$ double mutants and evaluated the thymol resistance of the double mutants in comparison with that of the corresponding single mutants. Both $c n a 1 \Delta$ ire $1 \Delta$ and $c n b 1 \Delta$ ire $1 \Delta$ exhibited similar levels of thymol resistance as the single mutants (Figure 3E), suggesting that deletion of both the calcineurin and UPR pathways does not provide additional or synergistic effects on thymol susceptibility.

\subsection{The Effect of Thymol on the Ergosterol Biosynthesis in C. neoformans}

The finding that the hog $1 \Delta$ mutant was sensitive to thymol treatment prompted us to test whether thymol induces Hog1 phosphorylation, because Hog1 is phosphorylated or dephosphorylated in response to external stresses in C. neoformans [20]. In support of the thymol-sensitivity of the hog1 $1 \Delta$ mutant, Hog1 was found to be dephosphorylated in the presence of thymol (Figure 4A and Figure S3A). The fact that the viability of Aspergillus flavus is decreased due to reactive oxygen species (ROS) generation induced by thymol treatment and that the Cryptococcus HOG pathway plays a critical role in oxidative stress led us to measure intracellular ROS levels in the presence of thymol [12,20]. In contrast to $A$. flavus, thymol treatment did not produce noticeable ROS in C. neoformans (Figure S3B) and did not induce ROS detoxification genes (Figure S3C).

One of the major roles of the Cryptococcus HOG MAPK pathway is the regulation of ergosterol biosynthesis. Three genes (HMG1 encoding HMG-CoA reductase, ERG1 encoding squalene epoxidase, and ERG11 encoding lanosterol 14- $\alpha$ demethylase) are known to be involved in the rate-limiting step of ergosterol biosynthesis [32]. We examined the expression levels of HMG1, ERG1, and ERG11 during thymol treatment [33]. We found that the expression levels of these three genes gradually decreased upon thymol treatment (Figure 4B). To determine whether thymol inhibits ergosterol biosynthesis in a HOG MAPK pathway-dependent manner, we examined intracellular ergosterol content 
in the WT and $\operatorname{hog} 1 \Delta$ mutants upon thymol treatment. Similar to a previous study [32], ergosterol content in the $\operatorname{hog} 1 \Delta$ mutant was higher than that in the WT. In agreement with the reduced expression levels of ergosterol biosynthetic genes, ergosterol content also reduced in the WT upon thymol treatment, while it did not change in the hog1 $\Delta$ mutant (Figure 4C). These results indicate that thymol negatively regulates ergosterol production in a HOG MAPK-dependent manner in C. neoformans.

A

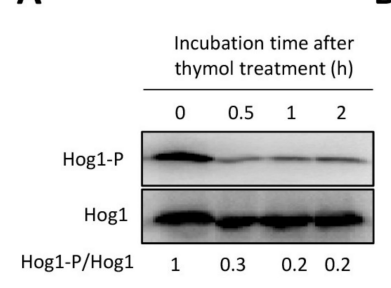

B

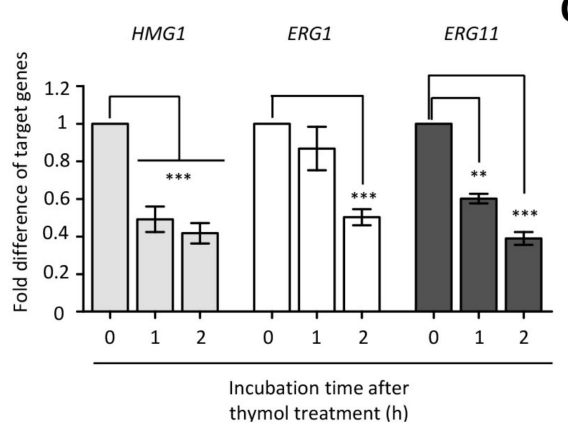

C

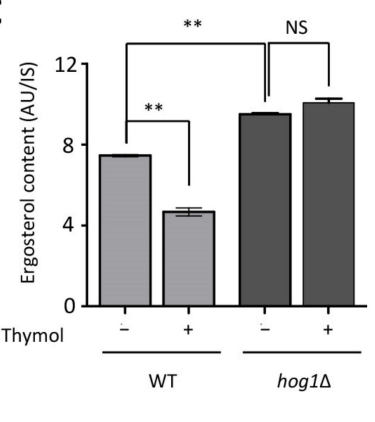

Figure 4. Thymol decreased cellular ergosterol content in a Hog1-dependent manner. (A) Thymol induced Hog1 dephosphorylation. Hog1 phosphorylation was detected using an anti-dually phosphorylated p38 antibody. Hog1 polyclonal antibody was used as a loading control. (B) Expression levels of gene involved in ergosterol biosynthesis reduced upon thymol treatment. cDNA was synthesized from total RNA obtained from the C. neoformans wild-type (H99) strain treated with or without thymol. For statistical analysis, the Bonferroni's multiple comparison test was performed using Prism software. (C) Change in cellular ergosterol measurement in the WT and hog1 $\Delta$ mutant, in the presence or absence of thymol. Relative ergosterol contents were calculated with $5 \alpha$-cholestan-3 $\beta$-ol as an internal control. For statistical analysis, experiments with three independent biological samples were executed. Error bars indicate S.E.M. Asterisks indicate statistical significance of difference ${ }^{*} p<0.05,{ }^{* *} p<0.01,{ }^{* * *} p<0.001$, and NS non-significant). AU, arbitrary unit.

To elucidate the relationship between calcineurin and HOG pathways with respect to thymol sensitivity, we constructed $c n a 1 \Delta$ hog $1 \Delta$ and $c n b 1 \Delta$ hog $1 \Delta$ double mutants and observed the growth of these double mutants in the presence of thymol. As expected, the cna1 $1 \Delta \circ g 1 \Delta$ and cnb1 $\operatorname{hog} 1 \Delta$ double mutants exhibited significant growth defects compared to the corresponding single mutants in the presence of thymol (Figure 5A). This result suggests that calcineurin and HOG pathways play redundant roles in conferring thymol resistance to $C$. neoformans. Low levels of sorbitol are known to enhance membrane stability in fungal protoplasts [34], and thymol is indirectly associated with membrane damage by reducing ergosterol biosynthesis in C. neoformans. Therefore, we investigated whether sorbitol treatment could restore thymol-induced growth suppression in the WT and mutant strains. Overall, C. neoformans WT and mutant strains exhibited growth enhancement in the presence of sorbitol $(0.05 \mathrm{M})$ even under thymol treatment (Figure $5 \mathrm{~B})$. Notably, restoration of the reduced growth upon thymol treatment was more evident in both cna1 $\operatorname{hog} 1 \Delta$ and cnb1 $\Delta$ hog $1 \Delta$ mutants in the presence of sorbitol $(0.05 \mathrm{M})$. However, this effect was not maintained in the presence of increased sorbitol concentration $(0.25 \mathrm{M})$, which showed more severe growth defects in all of the WT and mutant strains, as compared with those without sorbitol treatment (Figure 5B). These results indicate that the susceptibility of the hog $1 \Delta$ mutant to thymol is partly attributed to membrane instability, and that other cellular processes also cooperate in thymol resistance in a HOG MAPK-dependent manner. 


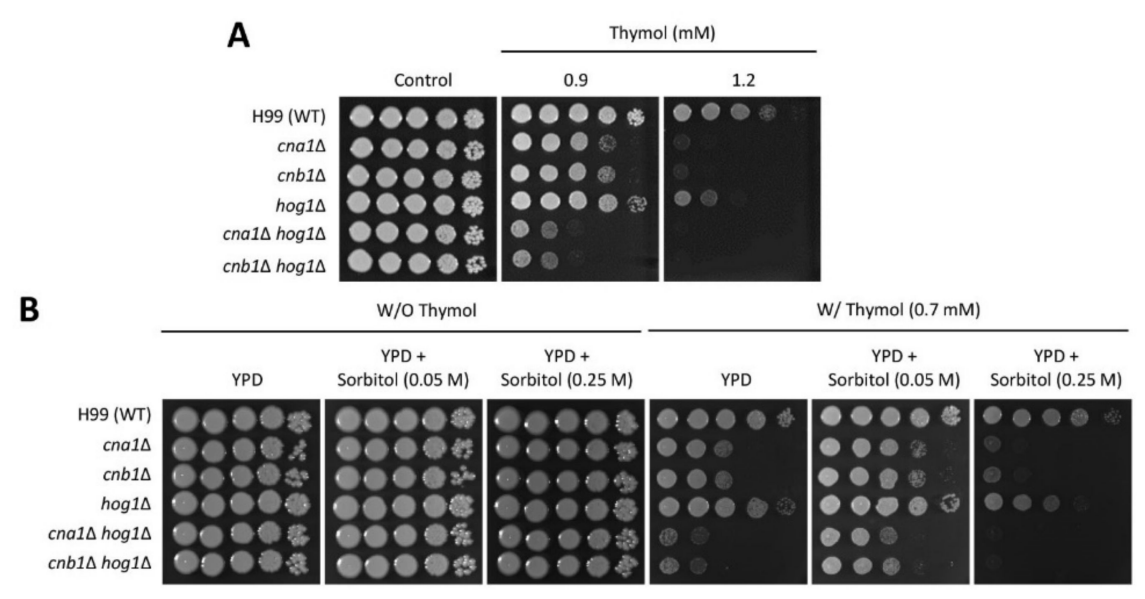

Figure 5. The redundant role of HOG and calcineurin pathways in thymol resistance. (A,B) Overnightcultured cells were serially diluted ( 1 to $\left.10^{4}\right)$ and spotted onto YPD media containing the indicated concentration of thymol with and without sorbitol $(0.05$ or $0.25 \mathrm{M})$. The cells were further incubated at $30^{\circ} \mathrm{C}$ for 4 days and photographed daily.

\section{Discussion}

With the rapid advancement of analytical methods, novel phytochemicals have been actively isolated and functionally characterized. Recently, the antimicrobial mechanisms of action of terpenoid compounds, such as carvacrol, have been investigated in model yeasts, S. cerevisiae and C. albicans. Rao et al. demonstrated that carvacrol disturbs ion $\left(\mathrm{Ca}^{2+}\right.$ and $\left.\mathrm{H}^{+}\right)$homeostasis, thereby decreasing cell viability [28]. Using chemical-genetic profiling, Chaillot et al. revealed that the UPR pathway is a crucial regulator of carvacrol tolerance [35]. Transcriptome analysis performed by Ansari et al. revealed that perillyl alcohol, a monoterpene alcohol, decreases the expression levels of the calcineurin signaling pathway, and that the $c n b 1 \Delta$ homozygous mutant is sensitive to perillyl alcohol [36]. In addition to common targets, including calcineurin and UPR pathways, terpenes and terpenoids interfere with ergosterol biosynthesis and membrane integrity [36,37]. In this study, we demonstrated that thymol has conserved intracellular targets similar to those of carvacrol and perillyl alcohol in yeasts and elucidated its antifungal mechanism through a genetics approach in C. neoformans. First, perturbation of calcineurin and UPR signaling pathways resulted in a loss of viability in response to thymol. However, the fact that only the ire $1 \Delta$ mutant, and not the $h x l 1 \Delta$ mutant, exhibited sensitivity to thymol in this study is in stark contrast to the previous finding that strains lacking IRE1 or HAC1, which is a functional orthologous gene to HXL1, displayed similar resistance to carvacrol [35]. This, in turn, indicates that the Cryptococcus UPR pathway is evolutionarily distinct, as compared to other fungal UPR pathways. Second, many studies have reported that terpene and terpenoid compounds affect microbial cellular membranes, and thymol reduces ergosterol content, but a mechanistic view of this finding remains elusive. We found that thymol reduced ergosterol content in C. neoformans by modulating the expression levels of genes involved in ergosterol biosynthesis, and this regulatory mechanism was dependent on the HOG MAPK pathway.

Although terpenoids and terpenes share similar intracellular targets between fungi, regulatory mechanisms in response to these molecules are divergent. It is well known that terpene or terpenoid treatments produce ROS, which elicit oxidative stress, thereby leading to cell death. For example, thymol treatment leads to the production of ROS and nitric oxide in A. flavus [12]. However, in this study, we provide several lines of evidence showing that the fungicidal action of thymol in C. neoformans is distinct from the conserved antifungal effect caused by ROS production in other fungi. Our results show that thymol did not induce intracellular ROS production. In accordance with this result, expression levels of oxidative stress-related genes, such as $S R X 1$, which is strongly induced in oxidative stress [38], were not significantly changed. The divergent effects caused by terpene or 
terpenoid compounds are often fungal species-specific. Geraniol treatment induces ROS accumulation in A. flavus but does not affect the production of ROS in A. ochraceus [39]. In addition to cellular responses to ROS, the regulation of intracellular $\mathrm{Ca}^{2+}$ levels in response to thymol is different among eukaryotes. In S. cerevisiae, carvacrol, thymol, and eugenol induce a rapid influx of $\mathrm{Ca}^{2+}$ from the extracellular medium [28]. However, we were not able to detect noticeable changes in intracellular $\mathrm{Ca}^{2+}$ levels in the presence of thymol in C. neoformans.

Intriguingly, we found that thymol affects protein glycosylation by modulating the expression of genes involved in $\mathrm{N}$-glycosylation and $O$-glycosylation. Supporting our results, the $S$. cerevisiae strain with a deletion of $C A X 4$, which encodes dolichyl pyrophosphate phosphatase in the $\mathrm{N}$-glycosylation process, showed growth defects in response to thymol, and glycosylation-related genes were downregulated upon carvacrol treatment in C. albicans $[35,40]$. In this state, it is unclear how thymol suppresses the expression levels of glycosylation-related genes, because there was an increase in the activated form of HXL1, which is a positive regulator of these genes, and deletion of HXL1 did not affect growth inhibition in response to thymol. Furthermore, it could not exclude that thymol might directly affect enzymes required for the attachment of $\mathrm{N}$-glycan to the residues of CnCpy1. We believe that detailed mechanisms of thymol on the $\mathrm{N}$-glycosylation process should be characterized in future studies. However, several studies have provided evidence that calcium homeostasis in the ER and Golgi is required for export of secretory proteins and protein folding. Deletion of PMR1, encoding high-affinity $\mathrm{Ca}^{2+} / \mathrm{Mn}^{2+}$ P-type ATPase, which is required for $\mathrm{Ca}^{2+} / \mathrm{Mn}^{2+}$ transport into the Golgi, induces defects in $\mathrm{N}$-linked glycosylation in the presence of $\mathrm{Ca}^{2+}$-enriched conditions [41]. Furthermore, Colinet et al. recently reported that $\mathrm{Gdt1}$, which is a Golgi-localized cation $/ \mathrm{Ca}^{2+}$ exchanger, is required for the glycosylation of Cpy and Gas1 under high concentrations of external calcium [42]. The results of the present study that, upon treatment with thymol, there was a decrease in the expression levels of calcium transporter genes in the ER and Golgi and $N$-glycosylation of Cpy, although the relative intracellular calcium levels did not change, indicate that thymol might induce $\mathrm{Ca}^{2+}$ imbalance in each organelle. Therefore, the relationship between intracellular $\mathrm{Ca}^{2+}$ imbalance caused upon thymol treatment and $\mathrm{N}$-linked and $\mathrm{O}$-linked glycosylation should be of interest.

In this study, we investigated the antifungal activity of the thymol in vitro system, not in vivo system, indicating that the efficacy of the thymol in vivo system should be checked in further study. Because it has been reported that cytotoxicity induced by thymol treatment occurs in diverse cell lines [43], it would be of our interest to modify the chemical structure of thymol aiming to reduce its cytotoxic effects.

Taken together, we suggest that thymol induces Hog1-dephosphorylation, thereby decreasing ergosterol biosynthesis by suppressing ERG1, ERG11, and HMG1 expression. Thymol resulted in intracellular calcium imbalance by regulating calcium transporter genes in a calcineurin-dependent and -independent manner. Furthermore, thymol affects protein glycosylation by controlling the expression of genes encoding $\mathrm{O}$-glycosylation and $\mathrm{N}$-glycosylation (Figure 6). Therefore, this study could improve our understanding of the molecular mechanisms of the fungal pathogen in the treatment of terpene compounds. 


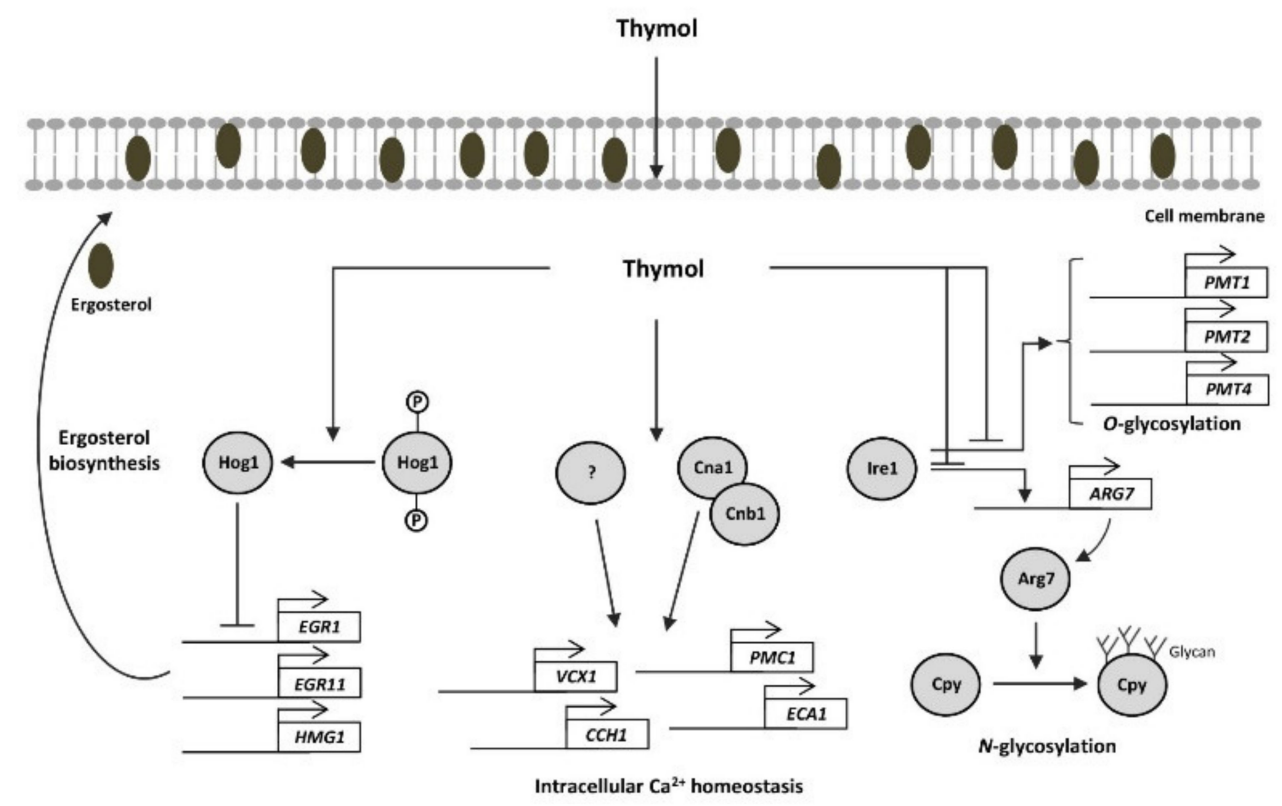

Figure 6. The suggested antifungal mechanism of thymol in C. neoformans. Thymol induced Hog1-dephosphorylation, thereby decreasing ergosterol biosynthesis. Thymol resulted in intracellular calcium imbalance by regulation of calcium transporter genes in calcineurin-dependent and -independent manners. Furthermore, thymol affects protein glycosylation by controlling expressions of genes encoding $\mathrm{O}$-glycosylation and $\mathrm{N}$-glycosylation.

\section{Materials and Methods}

\subsection{Strain and Media}

The strains used in this study are listed in Table S1. C. neoformans strains were cultured in yeast extract-peptone-dextrose (YPD) medium at $30^{\circ} \mathrm{C}$.

\subsection{Construction of C. neoformans Mutant Strains}

Deletion mutant strains were constructed in the C. neoformans serotype A H99S strain background using split marker/double-joint PCR strategies [19]. The genetic information for each gene was obtained from FungiDB (https: / / fungidb.org/fungidb/, accessed on 5 May 2018) The primers used in this study are described in Table S2. The gene deletion cassette and transformation were generated as previously described [19]. To confirm the genetic mutation of mutants, Southern blot analysis was performed using a gene-specific probe, as previously described [19].

\subsection{Total RNA Isolation, cDNA Synthesis, and Quantitative Reverse Transcription PCR}

An overnight culture of $C$. neoformans was inoculated into fresh YPD medium, such that the $\mathrm{OD}_{600}$ post inoculation was 0.2 , and further incubated at $30^{\circ} \mathrm{C}$ until the $\mathrm{OD}_{600}$ of the culture reached 0.8 . Cells were then treated with thymol (final concentration of $1 \mathrm{mM}$ ) and further incubated at $30{ }^{\circ} \mathrm{C}$ for the indicated time-points. The methods for total RNA isolation, RNA purification, and cDNA synthesis have been described previously [32]. We performed quantitative reverse transcription PCR analysis with the gene-specific primers listed in Table S2 using the CFX96 Real-Time PCR Detection System (Bio-Rad, Hercules, CA, USA). Relative expression of the target genes was determined using the $2^{-\Delta \Delta C t}$ method, and statistical analyses were performed using one-way analysis of variance with Bonferroni's multiple-comparison test (GraphPad Software Inc., San Diego, CA, USA). The Actin1 gene was used as an internal control for quantification of gene expression.

\subsection{Protein Extraction and Western Blot Analysis}

The Cpy- $4 \times$ FLAG culture was diluted in fresh YPD media such that the $\mathrm{OD}_{600}$ value post dilution was 0.2 , and further incubated at $30{ }^{\circ} \mathrm{C}$ until the $\mathrm{OD}_{600}$ reached 0.8 . Fifty 
milliliters of culture were pelleted for the zero-time sample. The remaining culture was treated with $1 \mathrm{mM}$ thymol and further incubated for the indicated time-points. Whole-cell lysates were extracted using lysis buffer, as previously described [44]. An anti-dually phosphorylated p38 antibody (catalog number 4511, Cell Signaling Technology, Danvers, MA, USA) was used to assess the Hog1 phosphorylation level, while anti- $\beta$-actin antibody (catalog number SC-47778, Santa Cruz Biotechnology., Dallas, TX, USA) and anti-polyclonal Hog1 antibody (catalog number SC-9079, Santa Cruz Biotechnology) were used as a loading control.

\subsection{Spotting Assay}

C. neoformans strains were cultured in liquid YPD medium overnight at $30^{\circ} \mathrm{C}$. The cells were 10 -fold serially diluted (1 to $10^{4}$ dilutions) in sterile distilled water and spotted onto solid YPD medium containing the indicated concentrations of terpene or terpenoid compounds. The cells were further incubated for 2-5 days and photographed daily.

\subsection{Determination of Relative Intracellular $\mathrm{Ca}^{2+}$ Levels}

Each C. neoformans strain was adjusted to $2 \times 10^{7}$ cells $/ \mathrm{mL}$ in $15 \mathrm{~mL}$ of YPD medium. Next, the cells were treated with a final concentration of $1 \mathrm{mM}$ thymol, $100 \mathrm{nM} \mathrm{CaCl}$, and both thymol and $\mathrm{CaCl}_{2}$, and further incubated for $2 \mathrm{~h}$ at $30{ }^{\circ} \mathrm{C}$. After fixation, the cells were incubated with $0.1 \mu \mathrm{M}$ FURA-2-AM (catalog number F1201, Invitrogen, Waltham, MA, USA) for $2 \mathrm{~h}$ at $37^{\circ} \mathrm{C}$. After incubation, the FURA-2-AM fluorescence intensity was determined at excitation wavelengths of 340 and $380 \mathrm{~nm}$ and an emission wavelength of $505 \mathrm{~nm}$ using a fluorescence spectrophotometer (Tecan, Männedorf, Switzerland). The relative intracellular $\mathrm{Ca}^{2+}$ levels were expressed as the ratio of fluorescence intensity (FI) obtained by excitation at 340 and $380 \mathrm{~nm}\left(\mathrm{FI}_{340 \mathrm{~nm}} / \mathrm{FI}_{380 \mathrm{~nm}}\right)$.

\subsection{Construction of Strain Containing CPY-FLAG}

The strain tagged with Cpy (CNAG_06640)-4×FLAG was generated as follows: the three PCR fragments (promoter and exon of CPY gene (CPYPE), $4 \times$ FLAG tag sequence $(4 \times$ FLAG), and terminator of $C P Y$ gene $(C P Y t))$ were amplified using the primers listed in Table S2. The amplified CPYPE fragment was cloned into pGEM-T-Easy (catalog number A3600, Promega) to generate pGEM-CPYPE (KWE70). The $4 \times$ FLAG-CPYt fragment was generated using overlap PCR with the primers J737 and J740 and $4 \times$ FLAG and CPYt fragments as templates. The $4 \times$ FLAG-CPYt fragment was cloned into pGEM-T-Easy to construct pGEM-4xFLAG-CPYt (KWE71). The BamHI-digested $4 \times$ FLAG-CPYt fragment was subcloned into pGEM-CPYPE to generate the plasmid pGEM-CPY-4 $\times$ FLAG (KWE73). Next, the NotI-digested CPY-4×FLAG fragment was subcloned into pJAF12 containing a NEO selection marker, to generate the plasmid pJAF12-CPY-4×FLAG (KWE81). The NsiI-digested pJAF12-CPY-4×FLAG was biolistically transformed into the H99S strain. The insertion of the fragment was initially screened using diagnostic PCR with the primers J737 and J740. Next, the proper production of Cpy- $4 \times$ FLAG was confirmed using western blot analysis with an anti-FLAG antibody (catalog number F3165, Sigma, Saint Louis, MO, USA).

\subsection{ROS Measurement Assay}

ROS levels were measured as described previously, with minor modifications [45]. The cells were adjusted to a concentration of $1 \times 10^{7}$ cells $/ \mathrm{mL}$, treated with thymol for $1 \mathrm{~h}$, and then fixed with formaldehyde solution. Next, the cells were incubated with $10 \mu \mathrm{M} \mathrm{H}_{2}$ DCFDA (catalog number D399, Invitrogen) for $2 \mathrm{~h}$. After incubation, cells were washed three times with phosphate buffered saline and fluorescence was measured using fluorometry (Infinite 200) with emission and excitation wavelengths of 488 and $520 \mathrm{~nm}$, respectively. Fluorescence intensity was normalized to cell density. Statistical analyses were performed using one-way analysis of variance with Bonferroni's multiple comparison test. 


\subsection{Measurement of Ergosterol Content}

The overnight culture was inoculated into fresh YPD media, such that the $\mathrm{OD}_{600}$ value post inoculation was 0.2 , and further incubated at $30^{\circ} \mathrm{C}$ until the $\mathrm{OD}_{600}$ reached a value of 1.0. The cell culture was then treated with thymol (final concentration, $1 \mathrm{mM}$ ) and further incubated for $2 \mathrm{~h}$. The control cells were treated with DMSO only. After incubation for the indicated time-points, the cells were collected, frozen in liquid nitrogen, and lyophilized overnight. The dried cells were ground with a mortar and pestle. Ergosterol content was extracted as previously described, with minor modifications. Briefly, $100 \mathrm{mg}$ of dried cells were homogenized with $4 \mathrm{~mL}$ of chloroform/methanol $(2: 1, v / v)$ containing $5 \alpha$-cholestan-3 $\beta$-ol as an internal standard. After removal of the solvent using a stream of $\mathrm{N}_{2}$ gas, the extract was dissolved in chloroform and then filtered using $0.2 \mu \mathrm{m}$ hydrophobic polytetrafluoroethylene. After removal of the solvent using a stream of $\mathrm{N}_{2}$ gas, the resultant pellet was saponified at $90^{\circ} \mathrm{C}$ for $1 \mathrm{~h}$ in $1 \mathrm{~mL} \mathrm{KOH}$ solution $(20 \%(w / v) \mathrm{KOH}$ in $80 \%(v / v)$ ethanol). After cooling to room temperature, $1 \mathrm{~mL}$ of distilled water was added to each sample. Diethyl ether $(2 \mathrm{~mL})$ was added to the sample, and the mixture was shaken vigorously. After centrifugation, the solvent layer (supernatant) was transferred to a fresh vial. Extraction with diethyl ether was performed three times, and the collected organic solvent layer was dried with $\mathrm{N}_{2}$. For the derivatization step, the dried samples were dissolved in chloroform, and an equal volume of chloroform containing $\mathrm{N}$-methyl$\mathrm{N}$-trimethylsilyltrifluoroacetamide was added and incubated at $80^{\circ} \mathrm{C}$ for $30 \mathrm{~min}$. After derivatization, the samples were dried with $\mathrm{N}_{2}$ and dissolved in chloroform. The dissolved samples were injected into a GC-MS 7890A/5975C system (Agilent, Santa Clara, CA, USA) equipped with a DB-5MS capillary column ( $30 \mathrm{~m} \times 0.25 \mathrm{~mm}$ ID, $0.25 \mu \mathrm{m}$ thickness, Agilent). The analytical conditions of GC-MS were as follows: electron impact, $70 \mathrm{eV}$; MS source temperature, $230^{\circ} \mathrm{C}$; injection temperature, $180^{\circ} \mathrm{C}$; carrier gas, helium; flow rate, $1 \mathrm{~mL} \cdot \mathrm{min}^{-1}$; column temperature program for ergosterol, $180^{\circ} \mathrm{C}$ for $5 \mathrm{~min}$, increased to $300{ }^{\circ} \mathrm{C}$ at $2{ }^{\circ} \mathrm{C} \mathrm{min}{ }^{-1}$ and held for $5 \mathrm{~min}$; and ergosterol was identified in a selected ion chromatogram at $m / z=468$ using authentic standards, as well as web-based libraries including Wiley 7th edition (https:/ / onlinelibrary.wiley.com, accessed on 12 June 2020), and the National Institute of Standards and Technology version 2.0, (https:/ / chemdata. nist.gov/mass-spc/ms-search, accessed on 12 June 2020). An authentic ergosterol standard (catalog number 45480, Sigma) was purchased and used for quantification.

\section{Conclusions}

The current study elucidated the antifungal mechanisms of thymol in C. neoformans using a reverse genetic approach. First, we found that thymol treatment affects intracellular calcium homeostasis by suppressing the expression of genes involved in the calcium transporters. Second, thymol treatment decreased expression levels of genes required for $\mathrm{N}$-glycosylation, thereby reducing protein glycosylation. Third, we demonstrated that thymol decreased ergosterol contents in a HOG pathway-dependent manner. In conclusion, this study provides insights into the potential use of thymol as an antifungal agent and advances our understanding of the antifungal mechanisms of action of thymol.

Supplementary Materials: The following are available online. Figure S1: Construction of gene deletion mutants, Figure S2: Expression levels of the UPR downstream genes, Figure S3: Thymol did not induce intracellular ROS levels in C. neoformans, Table S1: Strains used in this study, and Table S2: Primers used in this study.

Author Contributions: Conceptualization, K.-W.J. and S.L.; methodology and formal analysis, K.-W.J. and M.-S.C.; data curation, K.-W.J., B.-Y.C., H.-W.B., and M.-S.C.; original draft preparation, K.-W.J., B.-Y.C., H.-W.B., S.L., and M.-S.C.; and review and editing, K.-W.J. and S.L. All authors have read and agreed to the published version of the manuscript.

Funding: This work was supported by the Nuclear R\&D program of the Ministry of Science and Information and Communications Technologies (ICT) (Republic of Korea). 
Data Availability Statement: The data presented in this study are available in Supplementary information. Acknowledgments: We appreciate Yong-Sun Bahn at Yonsei University for providing the strains.

Conflicts of Interest: The authors declare no conflict of interest. The funders had no role in the design of the study, collection, analyses, or interpretation of data; in the writing of the manuscript; or in the decision to publish the results.

Sample Availability: Samples of the compounds and strains are available from the authors.

\section{References}

1. Bongomin, F.; Gago, S.; Oladele, R.O.; Denning, D.W. Global and Multi-National Prevalence of Fungal Diseases-Estimate Precision. J. Fungi 2017, 3, 57. [CrossRef] [PubMed]

2. Scorzoni, L.; de Paula, E.S.A.C.; Marcos, C.M.; Assato, P.A.; de Melo, W.C.; de Oliveira, H.C.; Costa-Orlandi, C.B.; Mendes-Giannini, M.J.; Fusco-Almeida, A.M. Antifungal Therapy: New Advances in the Understanding and Treatment of Mycosis. Front. Microbiol. 2017, 8, 36. [CrossRef] [PubMed]

3. Ostrosky-Zeichner, L.; Casadevall, A.; Galgiani, J.N.; Odds, F.C.; Rex, J.H. An insight into the antifungal pipeline: Selected new molecules and beyond. Nat. Rev. Drug Discov. 2010, 9, 719-727. [CrossRef] [PubMed]

4. Pfaller, M.A. Antifungal drug resistance: Mechanisms, epidemiology, and consequences for treatment. Am. J. Med. 2012, 125, S3-S13. [CrossRef] [PubMed]

5. VanEtten, H.D.; Mansfield, J.W.; Bailey, J.A.; Farmer, E.E. Two Classes of Plant Antibiotics: Phytoalexins versus "Phytoanticipins". Plant Cell 1994, 6, 1191-1192. [CrossRef] [PubMed]

6. Grayer, R.J.; Kokubun, T. Plant-fungal interactions: The search for phytoalexins and other antifungal compounds from higher plants. Phytochemistry 2001, 56, 253-263. [CrossRef]

7. Amiri, H. Essential oils composition and antioxidant properties of three Thymus species. Evid.-Based Complement. Altern. Med. eCAM 2012, 2012, 728065. [CrossRef] [PubMed]

8. Salehi, B.; Mishra, A.P.; Shukla, I.; Sharifi-Rad, M.; Contreras, M.D.M.; Segura-Carretero, A.; Fathi, H.; Nasrabadi, N.N.; Kobarfard, F.; Sharifi-Rad, J. Thymol, thyme, and other plant sources: Health and potential uses. Phytother. Res. PTR 2018, 32, 1688-1706. [CrossRef] [PubMed]

9. Nostro, A.; Blanco, A.R.; Cannatelli, M.A.; Enea, V.; Flamini, G.; Morelli, I.; Sudano Roccaro, A.; Alonzo, V. Susceptibility of methicillin-resistant staphylococci to oregano essential oil, carvacrol and thymol. FEMS Microbiol. Lett. 2004, 230, 191-195. [CrossRef]

10. Olasupo, N.A.; Fitzgerald, D.J.; Gasson, M.J.; Narbad, A. Activity of natural antimicrobial compounds against Escherichia coli and Salmonella enterica serovar typhimurium. Lett. Appl. Microbiol. 2003, 37, 448-451. [CrossRef]

11. Braga, P.C.; Alfieri, M.; Culici, M.; Dal Sasso, M. Inhibitory activity of thymol against the formation and viability of Candida albicans hyphae. Mycoses 2007, 50, 502-506. [CrossRef]

12. Shen, Q.; Zhou, W.; Li, H.; Hu, L.; Mo, H. ROS Involves the Fungicidal Actions of Thymol against Spores of Aspergillus flavus via the Induction of Nitric Oxide. PLoS ONE 2016, 11, e0155647. [CrossRef]

13. Zhang, J.; Ma, S.; Du, S.; Chen, S.; Sun, H. Antifungal activity of thymol and carvacrol against postharvest pathogens Botrytis cinerea. J. Food Sci. Technol. 2019, 56, 2611-2620. [CrossRef]

14. De Lira Mota, K.S.; de Oliveira Pereira, F.; de Oliveira, W.A.; Lima, I.O.; de Oliveira Lima, E. Antifungal activity of Thymus vulgaris L. essential oil and its constituent phytochemicals against Rhizopus oryzae: Interaction with ergosterol. Molecules 2012, 17, 14418-14433. [CrossRef]

15. Teixeira, A.P.C.; Nobrega, R.O.; Lima, E.O.; Araujo, W.O.; Lima, I.O. Antifungal activity study of the monoterpene thymol against Cryptococcus neoformans. Nat. Prod. Res. 2020, 34, 2630-2633. [CrossRef]

16. Rajasingham, R.; Smith, R.M.; Park, B.J.; Jarvis, J.N.; Govender, N.P.; Chiller, T.M.; Denning, D.W.; Loyse, A.; Boulware, D.R. Global burden of disease of HIV-associated cryptococcal meningitis: An updated analysis. Lancet Infect. Dis. 2017, 17, 873-881. [CrossRef]

17. Lin, X.; Heitman, J. The biology of the Cryptococcus neoformans species complex. Annu. Rev. Microbiol. 2006, 60, 69-105. [CrossRef]

18. Idnurm, A.; Bahn, Y.S.; Nielsen, K.; Lin, X.; Fraser, J.A.; Heitman, J. Deciphering the model pathogenic fungus Cryptococcus neoformans. Nat. Rev. Microbiol. 2005, 3, 753-764. [CrossRef]

19. Jung, K.W.; Lee, K.T.; So, Y.S.; Bahn, Y.S. Genetic Manipulation of Cryptococcus neoformans. Curr. Protoc. Microbiol. 2018, 50, e59. [CrossRef]

20. Bahn, Y.S.; Kojima, K.; Cox, G.M.; Heitman, J. Specialization of the HOG pathway and its impact on differentiation and virulence of Cryptococcus neoformans. Mol. Biol. Cell 2005, 16, 2285-2300. [CrossRef]

21. Kraus, P.R.; Fox, D.S.; Cox, G.M.; Heitman, J. The Cryptococcus neoformans MAP kinase Mpk1 regulates cell integrity in response to antifungal drugs and loss of calcineurin function. Mol. Microbiol. 2003, 48, 1377-1387. [CrossRef] [PubMed]

22. Odom, A.; Muir, S.; Lim, E.; Toffaletti, D.L.; Perfect, J.; Heitman, J. Calcineurin is required for virulence of Cryptococcus neoformans. EMBO J. 1997, 16, 2576-2589. [CrossRef] 
23. Cheon, S.A.; Jung, K.W.; Chen, Y.L.; Heitman, J.; Bahn, Y.S.; Kang, H.A. Unique evolution of the UPR pathway with a novel bZIP transcription factor, Hx11, for controlling pathogenicity of Cryptococcus neoformans. PLoS Pathog. 2011, 7, e1002177. [CrossRef] [PubMed]

24. Alspaugh, J.A.; Cavallo, L.M.; Perfect, J.R.; Heitman, J. RAS1 regulates filamentation, mating and growth at high temperature of Cryptococcus neoformans. Mol. Microbiol. 2000, 36, 352-365. [CrossRef] [PubMed]

25. Alspaugh, J.A.; Pukkila-Worley, R.; Harashima, T.; Cavallo, L.M.; Funnell, D.; Cox, G.M.; Perfect, J.R.; Kronstad, J.W.; Heitman, J. Adenylyl cyclase functions downstream of the $\mathrm{G} \alpha$ protein $\mathrm{Gpa} 1$ and controls mating and pathogenicity of Cryptococcus neoformans. Eukaryot. Cell 2002, 1, 75-84. [CrossRef] [PubMed]

26. Jung, K.W.; Yang, D.H.; Kim, M.K.; Seo, H.S.; Lim, S.; Bahn, Y.S. Unraveling Fungal Radiation Resistance Regulatory Networks through the Genome-Wide Transcriptome and Genetic Analyses of Cryptococcus neoformans. mBio 2016, 7. [CrossRef] [PubMed]

27. Jung, K.W.; Lee, Y.; Huh, E.Y.; Lee, S.C.; Lim, S.; Bahn, Y.S. Rad53- and Chk1-Dependent DNA Damage Response Pathways Cooperatively Promote Fungal Pathogenesis and Modulate Antifungal Drug Susceptibility. mBio 2019, 10. [CrossRef]

28. Rao, A.; Zhang, Y.; Muend, S.; Rao, R. Mechanism of antifungal activity of terpenoid phenols resembles calcium stress and inhibition of the TOR pathway. Antimicrob. Agents Chemother. 2010, 54, 5062-5069. [CrossRef]

29. Kmetzsch, L.; Staats, C.C.; Cupertino, J.B.; Fonseca, F.L.; Rodrigues, M.L.; Schrank, A.; Vainstein, M.H. The calcium transporter Pmc1 provides $\mathrm{Ca}^{2+}$ tolerance and influences the progression of murine cryptococcal infection. FEBS J. 2013, 280, $4853-4864$. [CrossRef]

30. Park, H.S.; Chow, E.W.; Fu, C.; Soderblom, E.J.; Moseley, M.A.; Heitman, J.; Cardenas, M.E. Calcineurin Targets Involved in Stress Survival and Fungal Virulence. PLoS Pathog. 2016, 12, e1005873. [CrossRef]

31. Winther, J.R.; Stevens, T.H.; Kielland-Brandt, M.C. Yeast carboxypeptidase Y requires glycosylation for efficient intracellular transport, but not for vacuolar sorting, in vivo stability, or activity. Eur. J. Biochem. 1991, 197, 681-689. [CrossRef]

32. Ko, Y.J.; Yu, Y.M.; Kim, G.B.; Lee, G.W.; Maeng, P.J.; Kim, S.S.; Floyd, A.; Heitman, J.; Bahn, Y.S. Remodeling of global transcription patterns of Cryptococcus neoformans genes mediated by the stress-activated HOG signaling pathways. Eukaryot. Cell 2009, 8, 1197-1217. [CrossRef]

33. Veen, M.; Stahl, U.; Lang, C. Combined overexpression of genes of the ergosterol biosynthetic pathway leads to accumulation of sterols in Saccharomyces cerevisiae. FEMS Yeast Res. 2003, 4, 87-95. [CrossRef]

34. Carrasco, H.; Raimondi, M.; Svetaz, L.; Di Liberto, M.; Rodriguez, M.V.; Espinoza, L.; Madrid, A.; Zacchino, S. Antifungal activity of eugenol analogues. Influence of different substituents and studies on mechanism of action. Molecules 2012, 17, 1002-1024. [CrossRef]

35. Chaillot, J.; Tebbji, F.; Remmal, A.; Boone, C.; Brown, G.W.; Bellaoui, M.; Sellam, A. The Monoterpene Carvacrol Generates Endoplasmic Reticulum Stress in the Pathogenic Fungus Candida albicans. Antimicrob. Agents Chemother. 2015, 59, 4584-4592. [CrossRef] [PubMed]

36. Ansari, M.A.; Fatima, Z.; Hameed, S. Anticandidal Effect and Mechanisms of Monoterpenoid, Perillyl Alcohol against Candida albicans. PLoS ONE 2016, 11, e0162465. [CrossRef]

37. Ahmad, A.; Khan, A.; Akhtar, F.; Yousuf, S.; Xess, I.; Khan, L.A.; Manzoor, N. Fungicidal activity of thymol and carvacrol by disrupting ergosterol biosynthesis and membrane integrity against Candida. Eur. J. Clin. Microbiol. Infect. Dis. 2011, 30, 41-50. [CrossRef]

38. Upadhya, R.; Kim, H.; Jung, K.W.; Park, G.; Lam, W.; Lodge, J.K.; Bahn, Y.S. Sulphiredoxin plays peroxiredoxin-dependent and -independent roles via the HOG signalling pathway in Cryptococcus neoformans and contributes to fungal virulence. Mol. Microbiol. 2013, 90, 630-648. [CrossRef]

39. Tang, X.; Shao, Y.L.; Tang, Y.J.; Zhou, W.W. Antifungal Activity of Essential oil Compounds (Geraniol and Citral) and Inhibitory Mechanisms on Grain Pathogens (Aspergillus flavus and Aspergillus ochraceus). Molecules 2018, 23, 2108. [CrossRef]

40. Darvishi, E.; Omidi, M.; Bushehri, A.A.; Golshani, A.; Smith, M.L. Thymol antifungal mode of action involves telomerase inhibition. Med. Mycol. 2013, 51, 826-834. [CrossRef]

41. Durr, G.; Strayle, J.; Plemper, R.; Elbs, S.; Klee, S.K.; Catty, P.; Wolf, D.H.; Rudolph, H.K. The medial-Golgi ion pump Pmr1 supplies the yeast secretory pathway with $\mathrm{Ca}^{2+}$ and $\mathrm{Mn}^{2+}$ required for glycosylation, sorting, and endoplasmic reticulum-associated protein degradation. Mol. Biol. Cell 1998, 9, 1149-1162. [CrossRef] [PubMed]

42. Colinet, A.S.; Sengottaiyan, P.; Deschamps, A.; Colsoul, M.L.; Thines, L.; Demaegd, D.; Duchene, M.C.; Foulquier, F.; Hols, P.; Morsomme, P. Yeast Gdt1 is a Golgi-localized calcium transporter required for stress-induced calcium signaling and protein glycosylation. Sci. Rep. 2016, 6, 24282. [CrossRef] [PubMed]

43. Gunes-Bayir, A.; Kocyigit, A.; Guler, E.M.; Dadak, A. In Vitro Hormetic Effect Investigation of Thymol on Human Fibroblast and Gastric Adenocarcinoma Cells. Molecules 2020, 25, 3270. [CrossRef] [PubMed]

44. So, Y.S.; Yang, D.H.; Jung, K.W.; Huh, W.K.; Bahn, Y.S. Molecular Characterization of Adenylyl Cyclase Complex Proteins Using Versatile Protein-Tagging Plasmid Systems in Cryptococcus neoformans. J. Microbiol. Biotechnol. 2017, 27, 357-364. [CrossRef]

45. Schneider Rde, O.; Diehl, C.; dos Santos, F.M.; Piffer, A.C.; Garcia, A.W.; Kulmann, M.I.; Schrank, A.; Kmetzsch, L.; Vainstein, M.H.; Staats, C.C. Effects of zinc transporters on Cryptococcus gattii virulence. Sci. Rep. 2015, 5, 10104. [CrossRef] 